\begin{tabular}{|c|c|c|}
\cline { 2 - 3 } & REVISTA SABERES APUDEP & Volumen 3 Número 2 \\
\hline
\end{tabular}

\title{
METODOLOGÍA 5'S Y SISTEMA DE PORTABILIDAD EN NOSOCOMIO GUANAJUATENSE, MÉXICANO
}

\section{5'S METHODOLOGY AND PORTABILITY SYSTEM IN NOSOCOMIO GUANAJUATENSE, MEXICANO}

\author{
Ramírez Chávez María Inés \\ Salud Pública del Estado de Guanajuato México \\ ramicha9@yahoo.com.mx \\ https://orcid.org/0000-0002-6625-931X \\ Bernardino Jesús Vázquez Fernández \\ Universidad Privada Domingo Savio Tarija, Bolivia. Centro de Investigación e Innovación \\ Tecnológica \\ Bernardino.Vasquezupds.edu.bo \\ https://orcid.org/0000-0002-9684-3533 \\ Jesús Ramón García Pérez \\ Universidad Tecnológica de Querétaro México \\ gapmkt@hotmail.com \\ Eduardo Modesto Vázquez Álvarez \\ Líder en salud publica nosocomial en el Estado de Guanajuato, México \\ episalva@yahoo.com.mx \\ https://orcid.org/0000-0001-6613-6523
}

\section{RESUMEN}

En este artículo se documentan los resultados de la aplicación de la metodología 5'S en un nosocomio de segundo nivel en Guanajuato Sur, con un marcado liderazgo en el proceso de gestión del Sistema de Portabilidad en el Estado de Guanajuato; bridando atención médica y hospitalaria a pacientes afiliados en entidades federativas como Nuevo León, Estado de México, Ciudad de México, Puebla, Hidalgo y Michoacán.

PALABRAS CLAVE Salud Pública; Portabilidad en Salud; Guanajuato México 


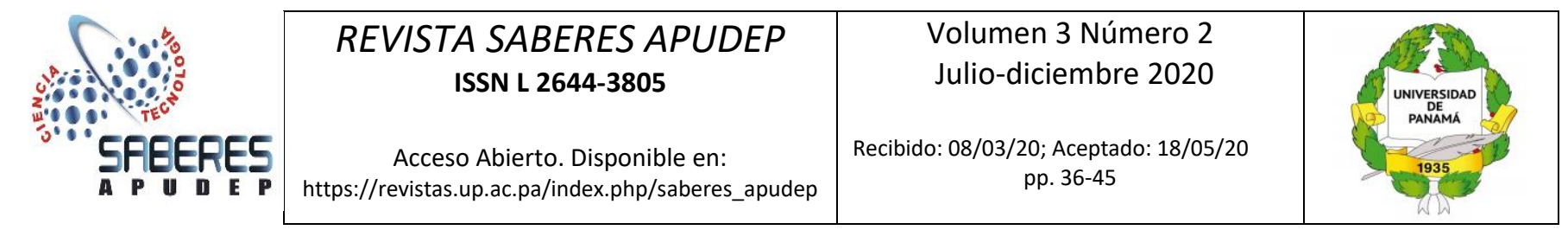

\begin{abstract}
This article documents the results of the application of the 5'S methodology in the finance department of Hospital Guanajuato Sur. With a marked leadership in the management process of the Portability System in the State of Guanajuato; offering hospital medical care to 6 states: Nuevo León, State of Mexico, Mexico City, Puebla, Hidalgo and Michoacán.
\end{abstract}

KEY WORDS Public health; Portability in Health; Guanajuato Mexico

\title{
INTRODUCCIÓN
}

Fundamentado en el Artículo 4 de la Constitución Política de los Estados Unidos Mexicanos "toda persona tiene derecho a la protección de la salud, La ley definirá las bases y modalidades para el acceso a los servicios de salud y establecerá la concurrencia de la Federación y las entidades federativas en materia de salubridad general", el sistema de salud en México ha transitado por varias reformas significativas, tratando de reducir las inequidades en la atención a la salud de la población (Secretaría de Salud, 2011).

La reforma más reciente, es la reforma a la Ley General de Salud del 2003, que da origen al Sistema de Protección Social en Salud (SPSS), cuyo brazo operativo es el Seguro Popular de Salud SPS (Gómez Dantes et ál., 2011). Este Programa está dirigido a la población sin ningún tipo de seguro público de salud como: autoempleados, desempleados, empleo informal; en la que se garantiza el acceso a alrededor de 260 intervenciones esenciales y 18 intervenciones de alto costo (Sistema de Protección en Salud, 2008, 2010).

La Estructura financiera del SPSS se basa en una lógica tripartita de derechos y responsabilidades: el gobierno federal, un contribuyente corresponsable y el beneficiario: en este caso el contribuyente corresponsable es el gobierno federal y las 


\begin{tabular}{|c|c|c|}
\cline { 2 - 3 } & REVISTA SABERES APUDEP & Volumen 3 Número 2 \\
\hline
\end{tabular}

entidades federativas que aportan por personas afiliadas, las necesidades de salud, el esfuerzo estatal y el desempeño, mientras que la cuota familiar se paga atendiendo a las condiciones socioeconómicas de la familia ubicadas en un estrato de ingreso (deciles). Los cuatro deciles más bajos no cubren esta cuota y pertenecen al régimen no contributivo (Frenk et ál.,2006).

Corresponde al Ejecutivo Federal, a través de la Secretaría de Salud, optimizar la utilización de sus instalaciones y compartir la prestación de servicios, así como definir las bases para la Compensación Económica Interestatal entre las entidades federativas, instituciones y establecimientos del Sistema Nacional de Salud, por concepto de prestación de servicios de salud, previa opinión de la Secretaría de Hacienda y Crédito Público.

El Programa Nacional de Portabilidad. - Es parte del Sistema de Protección Social en Salud (SPSS) y tiene como objetivo asegurar la prestación completa de servicios de salud al beneficiario sin importar la ubicación geográfica al momento de solicitar atención médica y su capacidad de pago; considerándose la Compensación Económica Interestatal como mecanismo de pago entre las entidades federativas por la prestación de servicios de salud brindados a los beneficiarios del Sistema fuera del área de circunscripción territorial en la que están afiliados (Ibarra et át., 2013)

Dicha Compensación Económica Interestatal se llevará a cabo conforme a lo establecido en la Norma Sanitaria NOM-004-SSA3-2012, que entre sus especificaciones señala:

- La colaboración y coordinación de las acciones entre los "servicios estatales de salud" firmantes, consiste en proporcionar las prestaciones, intervenciones y los servicios médicos considerados dentro del "CAUSES" vigente (Catálogo Universal de Servicios de Salud); definición explícita de cada una de las 


\begin{tabular}{|c|c|c|}
\cline { 2 - 3 } & REVISTA SABERES APUDEP & Volumen 3 Número 2 \\
\hline
\end{tabular}

intervenciones preventivas, diagnósticas, de tratamiento, hospitalización y cirugía a la que tienen derecho los beneficiarios.

- I) En que los servicios no sean proporcionados en la entidad donde reside el beneficiario, siempre que éste sea referido por personal de salud del estado donde resida de manera permanente a otra entidad federativa, de acuerdo con los lineamientos $y$ procedimientos establecidos de referencia $y$ contrarreferencia de pacientes entre las unidades médicas que se encuentran dentro de las redes de servicios del "SPSS".

- II) Los casos en los que la distancia o tiempo de traslado no garantice la oportunidad del tratamiento, siempre que esté debidamente documentada y justificada la situación

- III) El beneficiario se encuentre en tránsito en una entidad federativa diferente a la de su afiliación de origen.

- IV) El afiliado realice un cambio de domicilio temporal interestatal.

- V) Urgencia Médica

Sin los parámetros solicitados en la NOM-004-SSA3- 2012 no es posible que se efectué el pago correspondiente a las atenciones sanitarias. Este modelo de financiamiento ha implicado un cambio importante en los incentivos para los gobiernos estatales y los prestadores de servicios como es el caso del hospital de segundo nivel Guanajuato Sur que, al no contar con un soporte portable adecuado, los casos exitosos de casos atendidos, no podían ser reportados eficientemente, reflejando pérdidas de insumos, equipo, medicamentos, infraestructura, entre otros, para pacientes afiliados al seguro popular en el estado guanajuatense.

En este articulo tiene como propósito documentar el éxito alcanzado en Portabilidad alcanzado por el Nosocomio Guanajuatense Mexicano al incorporar la metodología 5's en la prestación de servicios médicos a pacientes afiliados a otros Estados Federativos. 


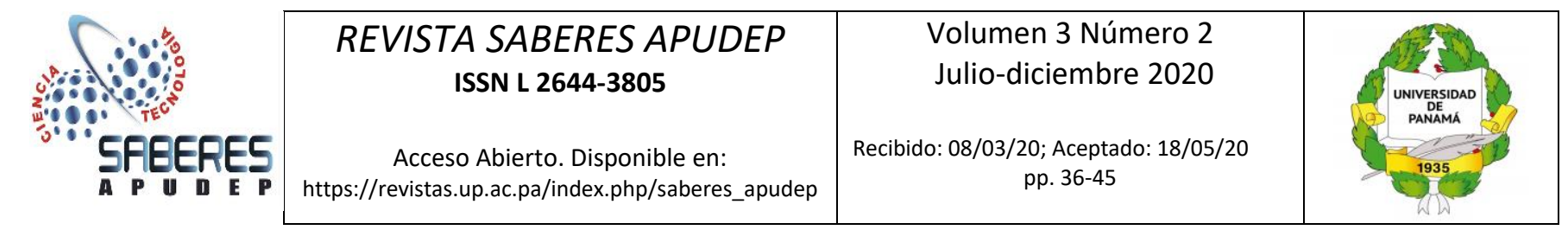

\section{MATERIALES Y MÉTODOS}

Se desarrollo una investigación con enfoque cualitativo, tipo Investigación acción reflexión, con alcance de transformar la situación inicial de Portabilidad de Salud Pública. Contemplando tres etapas: Diagnostica, Gestión y Evaluación.

\section{Etapa Diagnóstica}

Se examinaron los registros de Portabilidad en Salud del año 2015 al año 2017, para determinar que medidas establecidas en la normativa del expediente portable no se estaban cumpliendo, personal de salud involucrado, y los casos de asistencia médica hospitalaria admitidos durante el periodo en estudio.

\section{Etapa de Gestión}

Esta etapa se subdividió en fase de planeación, ejecución y seguimiento

En la Fase de Planeamiento se estructuro un cronograma de trabajo atendiendo a la Metodología 5's, una técnica de gestión japonesa basada en cinco principios básicos: clasificar, organizar, limpiar, estandarizar y diciplinar (Castillo, 2008), que permite implementar y establecer estándares para tener áreas y espacios de trabajo en orden y así con esto realizar eficientemente las actividades correspondientes.

Fase de ejecución contempló tres ejes fundamentales para portabilidad que son:

1.-Orientación al usuario: documentos que debe tener el usuario portable para ser beneficiario de la atención sanitaria en el nosocomio Guanajuato Sur.

2.- Formación del recurso humano nosocomial: capacitación continua sobre la norma sanitaria NOM-004-SSA3-2012. Comprometiéndoles a llevar a cabo su labor ya sea: medica, paramédica, administrativa o vigilancia conforme los estatutos brindados en portabilidad. Figura 1. 


\begin{tabular}{|c|c|c|}
\cline { 2 - 3 } & REVISTA SABERES APUDEP & Volumen 3 Número 2 \\
\hline
\end{tabular}

3. Sensibilización de los directivos: aportes y beneficios a partir de la gestión del Programa de Portabilidad de Salud.

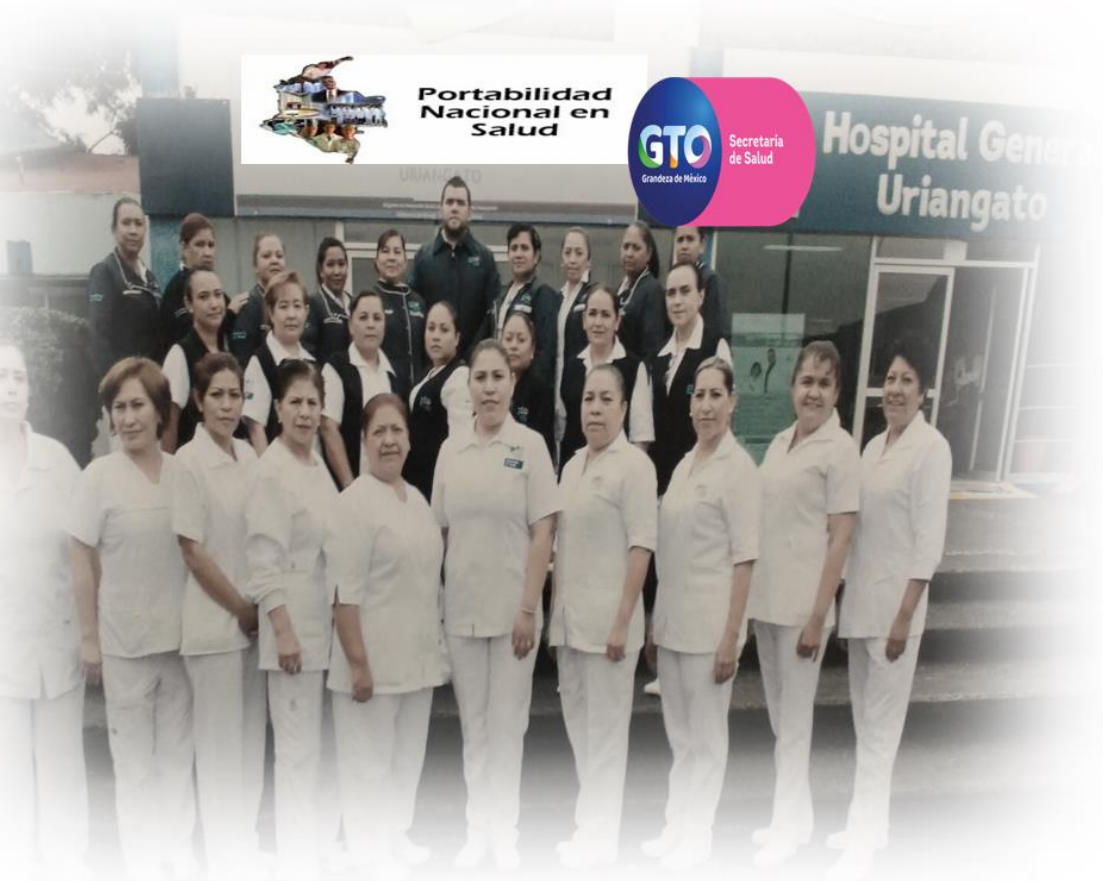

Figura 1. Personal de Salud. Guanajuato Sur. 2018

Fase de seguimiento se revisó cautelosamente los expedientes emitidos por el personal de salud y se encuestaba a los pacientes con expediente portable para determinar la satisfacción con los servicios recibidos.

Etapa de Evaluación se compararon los resultados obtenidos con el año 2017 y se presentó un informe técnico. 
Volumen 3 Número 2 Julio-diciembre 2020
Recibido: 08/03/20; Aceptado: 18/05/20 pp. 36-45

\section{RESULTADOS}

La Figura 2 muestra que para el año 2018 hubo un incremento de casos éxitos de Portabilidad en Salud del Hospital Guanajuato Sur, siendo los Entidades Federativas de Michoacán y Estado de México con mayor flujo de pacientes.

\begin{tabular}{|c|c|c|c|c|}
\hline Entidad atendida & Año 2017 & & Año 2018 & \\
\hline & $\begin{array}{l}\text { casos } \\
\text { atendidos }\end{array}$ & $\begin{array}{l}\text { Monto Portable } \\
\text { Recuperados }\end{array}$ & $\begin{array}{l}\text { casos } \\
\text { atendidos }\end{array}$ & Monto Portable \\
\hline Ciudad de México & 1 & $\$ 5,847.03$ & 1 & $\$ 1,332.60$ \\
\hline Colima & 1 & $\$ 8,720.89$ & & \\
\hline Estado de México & 2 & $\$ 26,914.44$ & 3 & $\$ 35,632.95$ \\
\hline Hidalgo & & & 1 & $\$ 8,339.07$ \\
\hline Michoacán & 297 & $\$ 2,281,555.61$ & 411 & $\$ 3,304,550.82$ \\
\hline Morelos & 1 & $\$ 10,624.82$ & & \\
\hline Nuevo León & & & 2 & $\$ 21,691.79$ \\
\hline Puebla & & & 2 & $\$ 13,360.01$ \\
\hline Zacatecas & 1 & $\$ 5,082.49$ & & \\
\hline Total & 303 & $\$ 2,338,745.28$ & 420 & $\$ 3,384,907.24$ \\
\hline
\end{tabular}

Figura 2. Entidades Federativas atendidas por portabilidad en el sur de Guanajuato

La Tabla 1 Muestran que en el año periodo del 2018 se atendieron 230 casos de atención nosocomial interestatal mexicana, con 106 casos válidos, 57 casos pagados y 3 casos no autorizados.

Tabla1 Estatus global de casos portable periodo enero-agosto2018

\begin{tabular}{|l|l|}
\hline Descripción & \# Casos \\
\hline Validados & 106 \\
\hline Autorizados & 5 \\
\hline No autorizados & 3 \\
\hline Pagados & 57 \\
\hline Pendientes & 59 \\
\hline Total & 230 \\
\hline
\end{tabular}




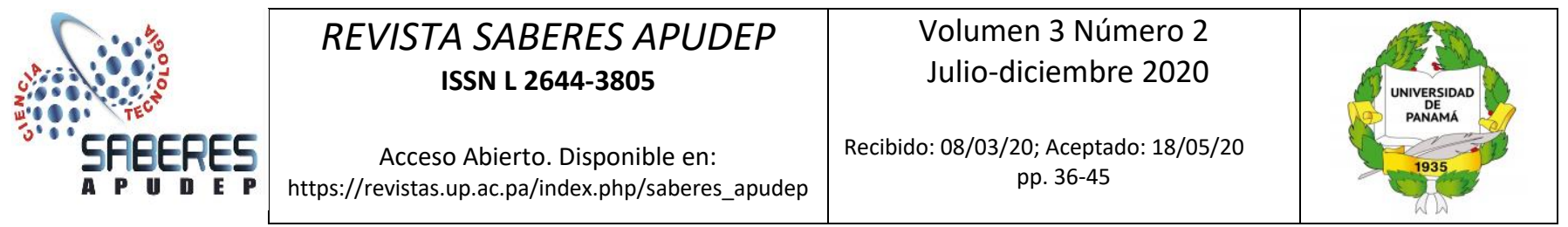

Los casos portable interestatales no autorizados han disminuido representando $1.30 \%$ de la actividad portable (Figura 3).

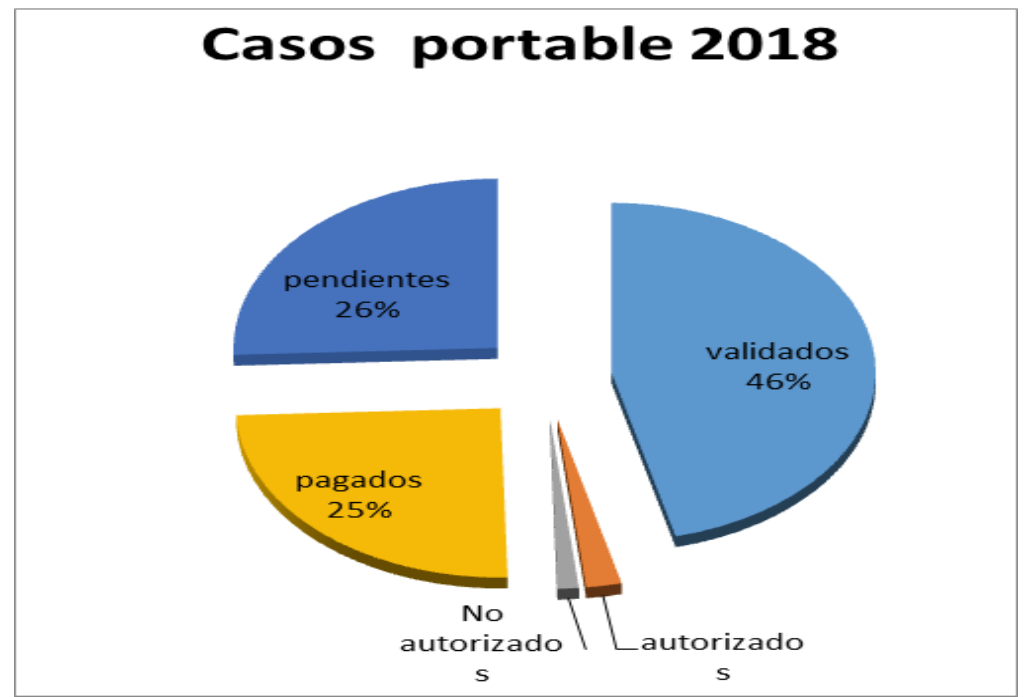

Figura 3.- Estatus global de casos portable periodo enero-agosto2018

En la cuestión de recuperación económica interestatal hospitalaria los montos comparados comienzan a ser visible los casos exitosos (Tabla 2).

Tabla 2 Montos nosocomiales recuperados en Hospital Guanajuato Sur

\begin{tabular}{|l|l|l|}
\hline Casos portables atendidos. & 2017 & 2018 \\
\hline $\begin{array}{l}\text { Total, anual Montos portables } \\
\text { recuperados }\end{array}$ & $\$ 2,338,745.28 \mathrm{MXP}$ & $\$ 3,384,907.24 \mathrm{MXP}$ \\
\hline $\begin{array}{l}\text { Total, anual Montos portables } \\
\text { NO recuperados }\end{array}$ & $\$ 96,862.08 \mathrm{MXP}$ & $\$ 18,672.25 \mathrm{MXP}$ \\
\hline Monto Anual portable & $\$ 2,435,607.36 \mathrm{MXP}$ & $\$ 3,403,579.49 \mathrm{MXP}$ \\
\hline
\end{tabular}




\begin{tabular}{|c|c|c|}
\cline { 2 - 3 } & REVISTA SABERES APUDEP & Volumen 3 Número 2 \\
\hline
\end{tabular}

Los montos no recuperados en el año 2018 fueron disminuidos en un $80.72 \%$ en comparativa del año 2017 por lo que el proceso holístico desarrollado en el hospital sur de Guanajuato es visible.

Para el periodo 2018, se presentaron tres quejas de servicios médicos prestados; se investiga tanto el servicio, como al profesional sanitario teniendo.

\section{CONCLUSIÓN}

La gestión de Portabilidad de casos interestatales en el Hospital Guanajuato Sur se ha consolidado, a través de la Metodología 5's; clasificar, ordenar los casos, entregar expedientes portables limpios, estandarizar la información y convertir estas acciones en hábito (disciplina), fundamentados en la Norma 004-SSA3-2012, permite aumentar los casos éxitos de portabilidad, mejorando la situación financiera, el acceso a insumos, medicamento, infraestructura y servicio medico a la población afiliada al Sistema de Protección Social de Salud del Estado de Guanajuato.

\section{REFERENCIAS BIBLIOGRÁFICAS}

Castillo, L. S. (2008). Metodología para la implementación de las 5’s. México: Asociación Nacional e instituciones de educación superior.

Frenk J., González-Pier E., Gómez-Dantés, O., Lezana M. A y Knaul F. (2006). Health System Reform in Mexico 1, Comprehensive reform to improve health system performance in Mexico. Lancet; 368:1524-1534.

Gómez Dantés, O., Sesma, S., Becerril, V., Knaul, F., Arreola, H y Frenk J. (2011). Sistema de salud de México. Salud Pública de México. 53 (2): 5220-5232

Ibarra, I., Martínez, G., Aguilera, N., Orozco, E., Fajardo-Dolci, G y González, Block, M. (2013). Salud Pública de México. 55 (3) 310-317

Norma Oficial Mexicana NOM-004-SSA3-2012, Del expediente clínico. http://dof.gob.mx/nota detalle popup.php?codigo=5272787 


\begin{tabular}{|c|c|c|}
\cline { 2 - 3 } & REVISTA SABERES APUDEP & Volumen 3 Número 2 \\
\hline
\end{tabular}

Secretaría de Salud. (2011). México: Hacia la integración funcional del Sistema Nacional de Salud. Secretaría de Salud: México DF.

Sistema de Protección Social en Salud. Informe de Resultados 2008. México, D.F.: Sistema de Protección Social en Salud, 2008.

Sistema de Protección Social en Salud. Informe de resultados enero-junio 2010. México: Comisión Nacional de Protección Social en Salud, Secretaría de Salud, 2010. 